\title{
Site-Selective Probe of Magnetic Excitations in Rare-Earth Nickelates Using Resonant Inelastic X-ray Scattering
}

\author{
Y. Lu, ${ }^{1}$ D. Betto, ${ }^{2}$ K. Fürsich, ${ }^{1}$ H. Suzuki, ${ }^{1}$ H.-H. Kim, ${ }^{1}$ G. Cristiani, ${ }^{1}$ G. Logvenov, ${ }^{1}$ N. B. Brookes, ${ }^{2}$ \\ E. Benckiser, ${ }^{1}$ M. W. Haverkort, ${ }^{3}$ G. Khaliullin, ${ }^{1}$ M. Le Tacon, ${ }^{4}$ M. Minola, ${ }^{1, *}$ and B. Keimer ${ }^{1, \dagger}$ \\ ${ }^{1}$ Max-Planck-Institut für Festkörperforschung, Heisenbergstrasse 1, 70569 Stuttgart, Germany \\ ${ }^{2}$ European Synchrotron Radiation Facility, 71 Avenue des Martyrs, Grenoble F-38043, France \\ ${ }^{3}$ Institut für Theoretische Physik, Universität Heidelberg, \\ Philosophenweg 19, 69120 Heidelberg, Germany \\ ${ }^{4}$ Karlsruher Institut für Technologie, Institut für Festkörperphysik, Hermann-v.-Helmholtz-Platz 1, \\ 76344 Eggenstein-Leopoldshafen, Germany
}

(Received 3 August 2017; revised manuscript received 20 June 2018; published 18 July 2018)

\begin{abstract}
We use high-resolution resonant inelastic x-ray scattering (RIXS) to study a thin film of $\mathrm{NdNiO}_{3}$, a compound whose unusual spin- and bond-ordered electronic ground state has been of long-standing interest. Below the magnetic ordering temperature, we observe well-defined collective magnon excitations along different high-symmetry directions in momentum space. The magnetic spectra depend strongly on the incident photon energy, which we attribute to RIXS coupling to different local electronic configurations of the expanded and compressed $\mathrm{NiO}_{6}$ octahedra in the bond-ordered state. Both the noncollinear magnetic ground state and the observed site-dependent magnon excitations are well described by a model that assumes strong competition between the antiferromagnetic superexchange and ferromagnetic doubleexchange interactions. Our study provides direct insight into the magnetic dynamics and exchange interactions of the rare-earth nickelates and demonstrates that RIXS can serve as a site-selective probe of magnetism in these and other materials.
\end{abstract}

DOI: 10.1103/PhysRevX.8.031014

Subject Areas: Condensed Matter Physics, Strongly Correlated Materials

\section{INTRODUCTION}

The spin ordering and dynamics in transition metal oxides are of long-standing interest from both fundamental and practical points of view [1,2]. In recent years, we have witnessed a surging effort in realizing a new generation of spintronic devices that may outperform the conventional electronic ones using magnetic oxides [3-5]. Many of these proposals are based on artificial heterostructures [6], or materials including multiple magnetic elements such as the double perovskites [7]. A key issue for the fundamental understanding of the correlated-electron physics at play in these complex materials, and consequently for a rational design and realization of oxide-based devices, is how to selectively probe the electronic and magnetic properties of the different functional components.

\footnotetext{
m.minola@fkf.mpg.de

b.keimer@fkf.mpg.de
}

Published by the American Physical Society under the terms of the Creative Commons Attribution 4.0 International license. Further distribution of this work must maintain attribution to the author(s) and the published article's title, journal citation, and DOI.
RIXS has recently emerged as a versatile tool for probing the ordering and dynamics of spin, charge, orbital, and lattice degrees of freedom in solids [8-15]. The chemical selectivity endowed by its resonant nature enables RIXS to individually address the properties that stem from different active elements, as well as their interactions in complex materials. In addition, the large cross section at x-ray resonance edges, combined with the high photon flux at modern synchrotron radiation sources, allows RIXS experiments on films and heterostructures with thickness down to a single unit cell $[16,17]$. Because of limitations in energy resolution, soft x-ray RIXS experiments have thus far mostly focused on cuprate compounds due to their exceptionally large magnon bandwidth. However, the latest advances in RIXS instrumentation now allow detection of collective magnetic excitations in a broader class of metal oxides [18].

In this article, we take advantage of the emerging highresolution soft X-ray RIXS capabilities to study the magnons in the perovskite rare-earth $(R)$ nickelates $R \mathrm{NiO}_{3}$, which exhibit an intriguing phase diagram controlled by the radius of $R$ [19-21]. Except for $\mathrm{LaNiO}_{3}$, bulk $R \mathrm{NiO}_{3}$ undergo a metal-insulator transition (MIT), accompanied by breathing-type lattice distortions forming a rock-salt 
pattern of expanded $\left(\mathrm{Ni}_{A}\right)$ and compressed $\left(\mathrm{Ni}_{B}\right) \mathrm{NiO}_{6}$ octahedra [21] [see Fig. 1(a)]. It was suggested [22] that this transition is an order-disorder transition of the $\mathrm{Ni}-\mathrm{O}$ bond-length fluctuations preexisting in the metallic phase. Concerning the nature of the MIT, various factors such as Fermi-surface nesting [23,24], Hund's coupling [25], and strong electron-lattice coupling [26-28] have been discussed. The particular importance of small or negative charge-transfer gap physics [29] for the bond disproportionation in bulk $R \mathrm{NiO}_{3}$ has been emphasized [30-35].

The magnetism of insulating $R \mathrm{NiO}_{3}$ is unusual. The $\mathrm{Ni}_{A(B)}$ sites with more ionic (covalent) $\mathrm{Ni}-\mathrm{O}$ bonds host spins of different sizes with $S_{A}>S_{B}$ [36]. At low temperatures, they condense into a four-sublattice noncollinear antiferromagnetic (AF) state with an ordering vector $\mathbf{Q}=$ $(1 / 4,1 / 4,1 / 4)$ (in pseudocubic notation). The nearestneighbor $S_{A}$ and $S_{B}$ spins are approximately orthogonal to each other [36-39], and the magnetic order can thus be viewed as a $90^{\circ}$ spin spiral. Such an ordering has no analogue in insulating oxides, and its underlying mechanism remains a puzzle. One of the main obstacles to understanding the magnetism of $R \mathrm{NiO}_{3}$ lies in the difficulties related to the characterization of different local spin states of the two inequivalent $\mathrm{Ni}$ sites and their associated exchange interactions. Although the two Ni sublattices are expected to respond differently in magnetic susceptibility [32], x-ray absorption [31], and optical spectroscopy [40] measurements, such predictions are difficult to test in practice, as these methods measure only the averaged properties of both sublattices.

In this work, we employ the unique site-selective capabilities of RIXS to probe the low-energy spin excitations associated with the two $\mathrm{Ni}$ sublattices in $\mathrm{NdNiO}_{3}$. Well-defined magnon dispersions along different highsymmetry directions in the first Brillouin zone were observed and reproduced by model calculations. After decades of research on the electronic phase behavior of $R \mathrm{NiO}_{3}$, these experiments can finally determine the magnetic exchange interactions and hence yield unprecedented insight into the mechanism driving the formation of the unusual noncollinear magnetic structure in this system.

\section{EXPERIMENTAL DETAILS AND RESULTS}

The RIXS experiment was performed at the ID32 beam line of the European Synchrotron Radiation Facility using the new ERIXS spectrometer [41]. We varied the scattering angle in the range from 55 to 135 degrees, which corresponds to momentum transfer of 0.4 to $0.8 \AA^{-1}$ at the $\mathrm{Ni} L_{3}$ edge around $853 \mathrm{eV}$. The combined instrumental energy resolution was set to about $50 \mathrm{meV}$ [full width at half maximum (FWHM)] to allow for reasonable acquisition time at sufficiently high resolving power. The incident photon polarization was kept parallel to the scattering plane. The $40-\mathrm{nm}$-thick $\mathrm{NdNiO}_{3}$ film was grown on a [001]-oriented $\mathrm{SrTiO}_{3}$ substrate by pulsed laser deposition.
It shows bulklike ordering behavior with transition temperature $T_{\mathrm{MIT}} \approx 200 \mathrm{~K}[39,42,43]$. The average $\mathrm{Ni}-\mathrm{O}$ bond disproportionation in the insulating phase was determined to be comparable to the value found in the bulk [42].

Figures 1(c) and 1(d) show the RIXS and electronyield x-ray absorption spectra (XAS) measured at the Ni $L_{3}$ edge $(2 p \rightarrow 3 d)$ on the $\mathrm{NdNiO}_{3}$ film at temperatures both below and above $T_{\mathrm{MIT}}$. The scattering geometry is shown in Fig. 1(b). The two sets of RIXS spectra shown in Fig. 1(c) were collected at momentum transfer $q_{111}=0.194$ [shorthand for $\mathbf{q}=0.194(1,1,1)]$, with the incident photon energy $E_{i}$ tuned to that of the two resonant peaks $A$ and $B$ around $E_{A} \approx 853 \mathrm{eV}$ and $E_{B} \approx 855 \mathrm{eV}$ in the XAS [Fig. 1(d)], respectively. Similarly to a previous study [44], the high-energy parts (with energy loss $\omega=$ $E_{i}-E_{f}>0.3 \mathrm{eV}$ ) of the two data sets are very different. Given that the RIXS intermediate states are the same as the

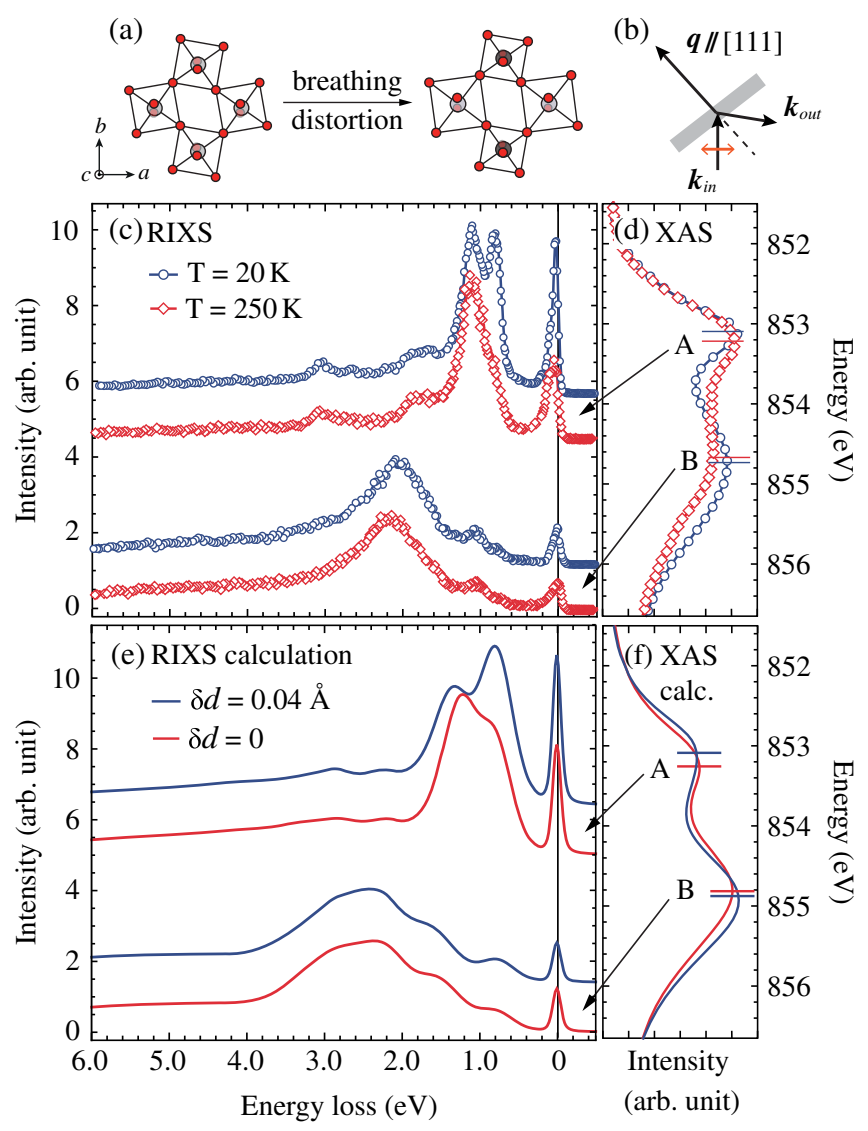

FIG. 1. (a) Sketch of the breathing lattice distortion across the MIT in the $R \mathrm{NiO}_{3}$. (b) The scattering geometry used in the experiment, where the red arrow indicates the electric vector of the photon polarization. (c) RIXS spectra and (d) XAS for the $\mathrm{NdNiO}_{3}$ film at temperatures below and above $T_{\text {MIT }}$. The RIXS spectra were collected at $q_{111}=0.194$ with incident photon energy tuned to the resonant peaks $A$ and $B$ marked by bars in panel (d). (e) RIXS spectra and (f) XAS calculated using a double-cluster model with $\delta d=0.04 \AA$ (blue) and $0 \AA$ (red) with the experimental geometry and light polarization in (c) and (d). 
XAS final states, it is helpful to examine the nature of the two peaks $A$ and $B$ in the XAS. As $R \mathrm{NiO}_{3}$ is believed to be "self-doped" $[45,46]$ with a ground-state configuration of predominantly $3 d^{8} \underline{L}$ character (where $\underline{L}$ denotes a ligand hole), the sharp peak $A$ can be assigned to the final state $2 p 3 d^{9} \underline{L}$ and the broad peak B to $2 p 3 d^{8}$, which correspond to excitations of $2 p$ core electrons into the $3 d$ and ligand states, respectively [31]. Based on this assignment, the decay process of RIXS with $E_{i}=E_{A}$ involves mostly transitions of the type $2 p 3 d^{9} \underline{L} \rightarrow\left(3 d^{8}\right)^{*} \underline{L}$, which give rise to large $d$ - $d$-excitation spectral weight centered around energy loss of $1 \mathrm{eV}$ [upper part of Fig. 1(c)]. For spectra measured with $E_{i}=E_{B}$, on the other hand, the core hole is annihilated largely by the ligand electrons, resulting in the broad fluorescence peak around $2-3 \mathrm{eV}$ [bottom part of Fig. 1(c)]. In both cases, the RIXS final states also include $3 d^{9} \underline{L}^{2}$ and associated particle-hole excitations, which generate a broad charge-transfer background. Figures 1(e) and 1(f) show the RIXS spectra and XAS calculated with multiplet ligand-field theory [47-49] using a double-cluster model [31]. The spectra calculated with bond disproportionation $\delta d$ set to $0 \AA(0.04 \AA$ [42]) correspond to those measured at $250 \mathrm{~K}(20 \mathrm{~K})$, respectively. At both temperatures, the main features discussed above are well captured by the calculation. The details are discussed in Appendix A.

From the above observations, it follows that, in the bonddisproportionated state with charge redistribution of the form $\left(3 d^{8} \underline{L}\right)\left(3 d^{8} \underline{L}\right) \rightarrow\left(3 d^{8}\right)_{A}\left(3 d^{8} \underline{L}^{2}\right)_{B}[30,31]$, the distinct local configurations of the $\mathrm{Ni}_{A}$ and $\mathrm{Ni}_{B}$ sites give rise to remarkably different energy profiles of their absorption spectra, as shown in Appendix A. For the $\mathrm{Ni}_{A}\left(\mathrm{Ni}_{B}\right)$ sites of majority $3 d^{8}\left(3 d^{8} \underline{L}^{2}\right)$ character, the XAS arises dominantly from excitations into the unoccupied $3 d$ (ligand) states, with spectral weight centered around energy $A(B)$ [Fig. 1(d)]. A similar site-dependent behavior is also seen for the x-ray magnetic circular dichroism (XMCD) [31]. By recalling that the coupling of low-energy magnon excitations to RIXS is controlled by XMCD [50], one realizes that the spin excitations associated with the two Ni sites can be individually probed by selectively tuning the incident photon energy to their respective resonances.

In the following, we focus on the RIXS spectra below $0.3 \mathrm{eV}$, which show clear asymmetric line shapes arising from low-lying excitations, as exemplified in Fig. 2(a). To extract the low-energy excitations, the elastic contribution can be removed by subtracting a Gaussian peak at $\omega=0$, with FWHM set to the experimental energy resolution of about $50 \mathrm{meV}$. The subtraction is performed for all the spectra presented hereafter. The raw spectra and the subtraction procedure can be found in Ref. [43]. Figure 2(b) shows an overview of the RIXS spectra measured at $20 \mathrm{~K}$ along the [111] momentum-transfer direction. The incident photon energy is tuned to $E_{i}=E_{A}$, which is expected to couple dominantly to the spins of $\mathrm{Ni}_{A}$ sites. The $\mathbf{q}$ values range from 0.40 to $0.65 \AA^{-1}$ in steps of $0.05 \AA^{-1}$, which correspond to
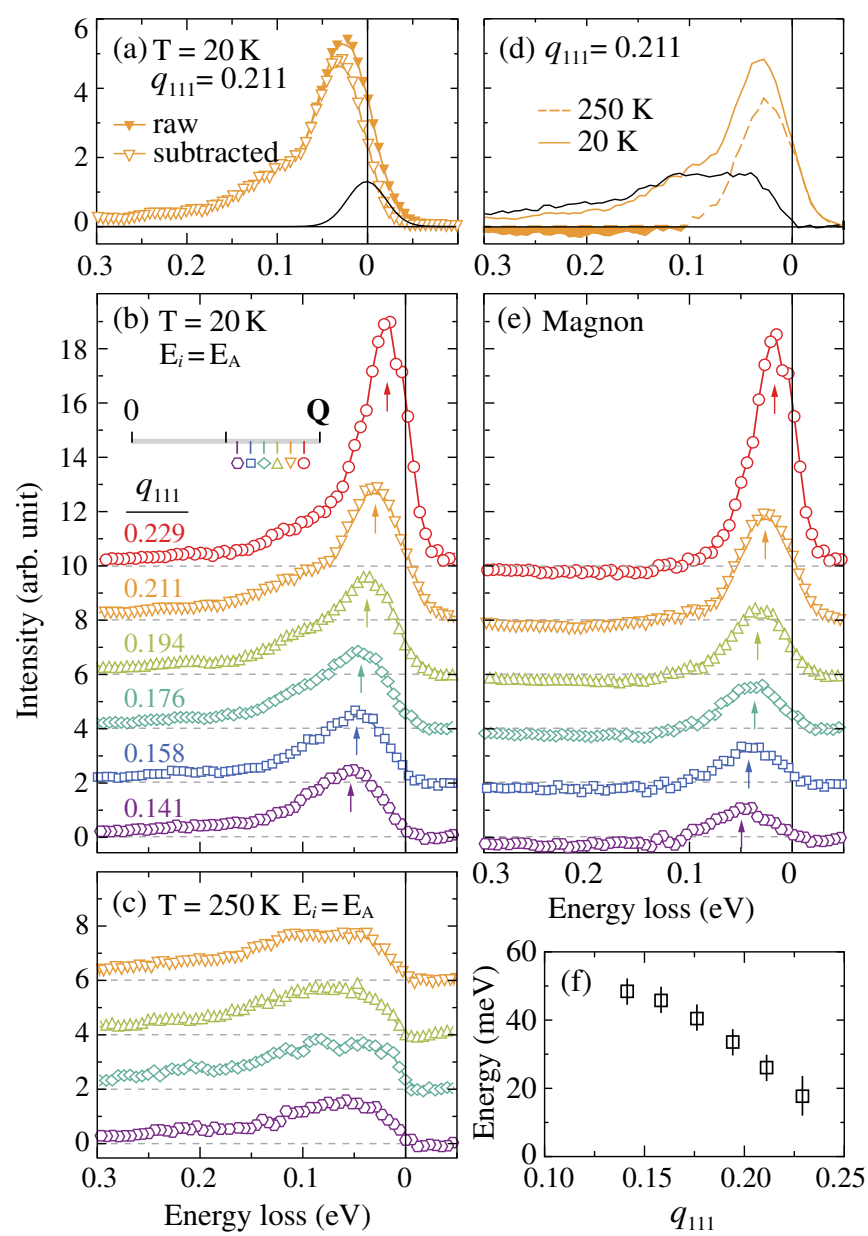

FIG. 2. (a) An example of the low-energy RIXS spectra of the $\mathrm{NdNiO}_{3}$ film measured with $E_{i}=E_{A}$ at $q_{111}=0.211$ at $20 \mathrm{~K}$. The elastic contribution is fitted by a Gaussian profile at $\omega=0$ with FWHM of about $50 \mathrm{meV}$. (b,c) Spectra at different $q_{111}$ values after subtracting the elastic part at (b) $20 \mathrm{~K}$ and (c) $250 \mathrm{~K}$. The inset in panel (b) shows the momentum transfer $\mathbf{q}$ of the spectra with respect to the ordering vector $Q_{111}=0.25$. (d) The magnon scattering intensity can be obtained by subtracting the nondispersing contribution (c) from the inelastic spectra in panel (b), as illustrated at $q_{111}=0.211$. The resulting spectra at all measured $q_{111}$ values are shown in panel (e). (f) The magnon dispersion obtained from panel (e).

$q_{111}=0.141-0.229$. A clear inelastic contribution peaked at $\omega \lesssim 50 \mathrm{meV}$ can be observed at all measured $\mathbf{q}$ values.

We employ several complementary diagnostic indicators to discriminate between magnon and phonon contributions to the inelastic intensity. First, as magnons become heavily overdamped (and hence nearly invisible to scattering probes) in the paramagnetic state, we compare RIXS spectra well above [Fig. 2(c)] and well below [Fig. 2(b)] the antiferromagnetic ordering temperature. In the paramagnetic state, the spectra exhibit no noticeable $\mathbf{q}$ dependence, as expected for optical phonons [51,52]. In addition, multimagnons [53] and charge excitations [51] may also contribute to these featureless spectra [54]. In contrast, the 
spectra in the AF state exhibit a prominent, strongly dispersive feature [indicated by arrows in Fig. 2(b)]. The dispersion emanates from the AF ordering wave vector $\mathbf{Q}$ and exhibits maxima at the borders of the AF Brillouin zone, as expected for collective excitations of the AF state. In addition, the intensity is maximal at $\mathbf{Q}$ and minimal at the AF Brillouin zone border, again supporting the assignment to magnons. The collective nature of the magnetic excitation is further verified by the independence of its energy on variation of $E_{i}$ around $E_{A}$ [43]. We emphasize that the unique energy selectivity of RIXS allowed us to maximize the cross section of local excitations by utilizing its relatively large energy separation from the continuum excitations in the RIXS intermediate state.

We proceed to extract the magnetic RIXS spectra by subtracting the nondispersing background measured at $250 \mathrm{~K}$ from the spectra at $20 \mathrm{~K}$, as illustrated in Fig. 2(d) for $q_{111}=0.211$. We notice the presence of a small negative intensity at energies above $0.1 \mathrm{eV}$ in the subtracted spectra: This is most likely the result of an additional particle-hole continuum emerging in the high-temperature metallic phase. Figure 2(e) shows the magnetic RIXS spectra at each $q_{111}$ after the subtraction. The magnon excitation energies are obtained from the peak positions of the resultant spectra and plotted in Fig. 2(f), where they exhibit a maximum of about $50 \mathrm{meV}$ at $q_{111}=0.141 \approx \mathbf{Q} / 2$. This energy scale of the magnetic dynamics is also confirmed by spectra measured along the [101] momentum-transfer direction [43].

Now we turn to the low-energy spectra measured with $E_{i}=E_{B}$, i.e., the energy that maximizes the RIXS coupling to the magnon excitations associated with the $\mathrm{Ni}_{B}$ sites. Figures 3(a) and 3(b) show the spectra measured along [111] at $20 \mathrm{~K}$ and $250 \mathrm{~K}$, respectively [55]. Contrary to the data taken with $E_{i}=E_{A}$, where the RIXS signal gains significant weight from collective magnon excitations, the spectra here are dominated by the nondispersing contribution. Following the same procedure as in Fig. 2(d), we

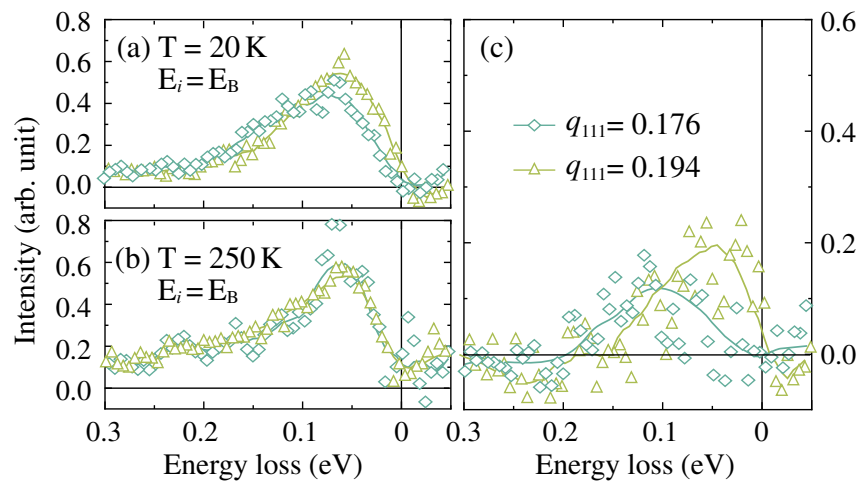

FIG. 3. Low-energy part of the RIXS spectra measured with $E_{i}=E_{B}$ at (a) $20 \mathrm{~K}$ and (b) $250 \mathrm{~K}$ after the subtraction of the elastic contribution. Panel (c) shows the magnon scattering intensity obtained by using the same procedure as in Fig. 2(c). The lines are a guide to the eye. subtract the $250 \mathrm{~K}$ spectra from the $20 \mathrm{~K}$ ones and plot in Fig. 3(c) the resulting spectra that one can assign to magnon excitations. While the remaining spectral weight may indicate a small contribution from dispersive magnons, the signal is too weak to be clearly distinguished from the statistical noise. The well-defined dispersing magnon excitations observed at $E_{i}=E_{A}$ and the nearly null observation at $E_{i}=E_{B}$ are in line with the prediction of the site-resolved spin susceptibilities [32].

\section{MAGNETIC RIXS CALCULATION}

The combined knowledge of the static AF ordering and the collective excitations of the $\mathrm{Ni}$ moments provides a solid basis-and simultaneously imposes stringent constraints-for low-energy spin models aimed to describe the hitherto unexplained noncollinear magnetic structure of $R \mathrm{NiO}_{3}$ [37-39]. Figure 4(a) shows a top view of the magnetic structure in $R \mathrm{NiO}_{3}$, where ferromagnetic (111) planes form a noncollinear $(\uparrow \leftarrow \downarrow \rightarrow)$ AF order. The ordering pattern of spins $S_{A}\left(S_{B}\right)$ residing on the $\mathrm{Ni}_{A}$ $\left(\mathrm{Ni}_{B}\right)$ sublattice readily follows from AF superexchange (SE) interactions $J_{2}$ and $J_{4}$, provided that $J_{4}>J_{2} / 2$. The latter condition can be justified by the large $p d \sigma$ chargetransfer fluctuations along the straight $\mathrm{Ni}-\mathrm{O}-\mathrm{Ni}-\mathrm{O}-\mathrm{Ni}$ bonds associated with $J_{4}$ [56]. However, the major question is which mechanism stabilizes the $90^{\circ}$ mutual orientation of the $S_{A}$ and $S_{B}$ sublattices, given that a conventional (Heisenberg) coupling $\left(\mathbf{S}_{A} \mathbf{S}_{B}\right)$ is frustrated and would result (via the "order-by-disorder" mechanism) in a collinear "up-up-down-down" arrangement instead.
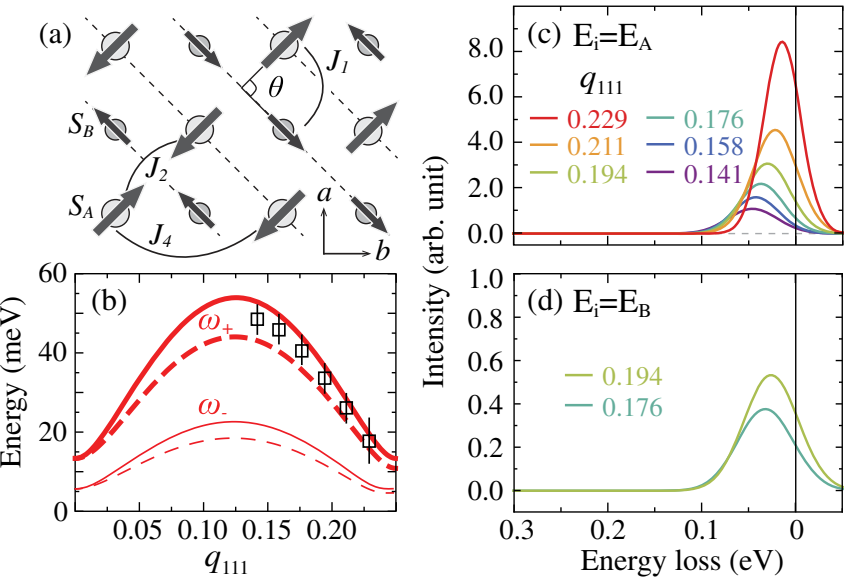

FIG. 4. (a) Schematic of the magnetic structure of $\mathrm{RNiO}_{3}$. It can be viewed as stacking of alternating ferromagnetic $S_{A}$ and $S_{B}$ planes along [111], as indicated by the dashed lines. (b) Calculated spin-wave dispersion along [111] for two kinds of domains (solid lines for $h k l$ and $\bar{h} \bar{k} l$, and dashed for $h \bar{k} l$ and $\bar{h} k l$ ). Each includes two branches (thick and thin) due to the disproportionated spins. Open symbols are experimental data from Fig. 2(f). (c,d) Calculated RIXS spectra of magnon excitations for the experimental geometry at $E_{i}=E_{A}$ and $E_{B}$. 
We suggest that, near the MIT, low-energy charge fluctuations between $\mathrm{Ni}_{A}$ and $\mathrm{Ni}_{B}$ sites with unequal spins $S_{A}>S_{B}$ lead to a double-exchange (DE) process that depends on the angle $\theta$ between $S_{A}$ and $S_{B}$ as $-\cos (\theta / 2)$ [57-59]. Competition between ferromagnetic $\mathrm{DE}$ and $\mathrm{AF}$ superexchange is known to result in noncollinear ordering [59], as recently observed in ferrates [56]. In the present context, the DE energy gain from the "left" and "right" $A B$ bonds in each direction [Fig. 4(a)] is proportional to $-\{[\cos (\theta / 2)]+\cos [(\pi-\theta) / 2]\}$, which is optimized at $\theta=\pi / 2$ and thus supports an unusual $90^{\circ}$ alignment of $S_{A}$ and $S_{B}$.

As far as small fluctuations around the classical spin pattern are concerned, one can incorporate the DE energy into a spin-only model $[56,60]$ with an effective parameter $J_{1}=J_{\mathrm{SE}}-J_{\mathrm{DE}}$ comprising both SE and DE contributions, where $J_{\mathrm{DE}}$ is associated with the kinetic energy due to lowenergy charge fluctuations between $\mathrm{Ni}_{A}$ and $\mathrm{Ni}_{B}$ sites. We calculate the magnon dispersions using the $J_{1}-J_{2}-J_{4}$ model, including also a single-ion anisotropy term $K$, assuming easy axes parallel to the cubic body diagonals. The spin disproportionation, quantified as $S_{A(B)}=\frac{1}{2}(1 \pm \delta)$, results in two magnon branches, $\omega_{+}$and $\omega_{-}$, with the former one carrying most of the scattering intensity. The disproportionation parameter $\delta=0.4$ was chosen such that it gives $S_{A} / S_{B} \approx 2$, consistent with experiment [36] and with the prediction of the double-cluster model for the experimental value of the bond disproportionation [31] (Fig. 1 and associated discussion). Details of the calculations are presented in Appendix B.

The spin Hamiltonian illustrated by Fig. 4(a) contains four adjustable parameters: $J_{1}, J_{2}, J_{4}$, and $K$. We note that the major magnon branch $\omega_{+}$is not sensitively dependent on the exact value of $J_{1}$. An accurate determination of $J_{1}$ requires resolution of the less intense, lower-energy branch $\omega_{-}$, which is beyond the capabilities of the current setup. However, the experimentally observed commensurate ordering wave vector implies the constraint $\left|J_{1}\right|<J_{1 \mathrm{c}}=$ $2 \sqrt{2 K\left(J_{2}+J_{4}\right) / 3}$ (Appendix B). In the fitting procedure, $J_{1}$ is therefore varied systematically between its upper and lower bounds to establish systematic errors for $J_{2}, J_{4}$, and $K$. Note that the maximum and minimum of $\omega_{+}$along the [111] direction and its maximum along the [101] direction [43] constitute three well-defined, independent pieces of experimental information that allow us to obtain these three parameters with good accuracy. The additional data points at different wave vectors further increase the confidence in these parameters.

Together with the statistical errors arising from the experimental uncertainty in determining the magnon energies, we obtain the following values and errors for the fitting parameters: $J_{2}=4.1 \pm 1.7, J_{4}=8.3 \pm 2.2$, and $K=1.3 \pm 1.3 \mathrm{meV}$, which implies $J_{1 \mathrm{c}}=6.3 \mathrm{meV}$. Figure 4(b) shows the magnon dispersions calculated with the best-fit parameters and $J_{1}=J_{1 \mathrm{c}} / 2$. The fitted value of the anisotropy constant $K$ results in a magnon gap of $13 \mathrm{meV}$ for the main branch of the dispersion, which is comparable to that observed in $\mathrm{La}_{2} \mathrm{NiO}_{4}$ [61]. However, the magnetocrystalline anisotropy is immaterial for our model and interpretation, which addresses the hierarchy of exchange interactions between the $\mathrm{Ni}$ ions. We emphasize once more that the dominance of the long-range interactions, $J_{2}$ and $J_{4}$, is a hallmark of the competition between superexchange and double-exchange interactions near the MIT, which greatly reduces the nearest-neighbor interaction $J_{1}$.

To calculate the magnetic spectra probed by RIXS, we use the RIXS operator [50] $R_{A(B)}=\sigma_{A(B)}^{(1)}\left(\boldsymbol{\epsilon}_{i} \times \boldsymbol{\epsilon}_{o}^{*}\right) \cdot \mathbf{S}_{A(B)}$, with $\boldsymbol{\epsilon}_{i(o)}$ the polarization of the incident (outgoing) photons. Here, $\sigma_{A(B)}^{(1)}$ are the calculated site-resolved XMCD spectra for the $\mathrm{Ni}_{A}\left(\mathrm{Ni}_{B}\right)$ sites. The calculated magnetic RIXS spectra for $E_{i}=E_{A}$ and $E_{B}$ are plotted in Figs. 4(c) and 4(d), respectively. All the spectra are averaged over contributions from the four $h k$ domains and then scaled with a universal factor to match the experimental intensity. For incident photon energy $E_{i}=E_{A}$, excellent agreement is achieved between calculation and experiment for the energy and spectral weight of the magnon excitation at all measured $\mathbf{q}$ values (including $\mathbf{q} \|$ [101] [43]), providing strong support for the proposed spin model. For $E_{B}$, while both calculation and experiment show strongly suppressed magnon spectral weight, the calculation shows a small deviation from the measurement, which could be attributed to limitations of the experimental sensitivity and/or to quantum effects beyond the linear-spin-wave approximation. While a state with nonzero moments on site $A$ only cannot be rigorously ruled out, the RIXS results and their theoretical description are quantitatively consistent with a noncollinear magnetic state with different but nonzero moments on sites $A$ and $B$.

\section{SUMMARY}

In summary, we have studied the electronic and magnetic excitations in $\mathrm{NdNiO}_{3}$ with RIXS at the Ni $L_{3}$ edge. The observed complex energy dependence of the RIXS spectra is attributed to the different local configurations and spin states in the bond-disproportionated state and their consequent distinct coupling to the RIXS process. The observed magnetic order and excitations are well captured by an effective spin model based on strong competition between superexchange and double-exchange interactions in $\mathrm{RNiO}_{3}$. Our study demonstrates the capability of RIXS to determine site-selective contributions to the collective magnetic dynamics, and it provides an example for its application in oxide thin films and heterostructures that contain several functional materials and/or inequivalent structural sites. With the development of future x-ray sources and other high-resolution RIXS setups, such as the one that was recently installed at beam line I21 of the Diamond Light Source [62], we expect that these advances will come to full fruition. 


\section{ACKNOWLEDGMENTS}

We are grateful to G. Ghiringhelli and L. Braicovich for facilitating the RIXS measurements using the ERIXS spectrometer. We thank E. Lefrançois for assistance at the ID32 beam line. We thank the Deutsche Forschungsgemeinschaft (DFG) for financial support under Grant No. TRR 80, Project No. G1.

Y. L. and D. B. contributed equally to this work.

\section{APPENDIX A: XAS AND RIXS SPECTRA CALCULATED USING A DOUBLE-CLUSTER MODEL}

The XAS and RIXS spectra shown in Figs. 1(e) and 1(f) were calculated using a double-cluster model introduced in Ref. [31], which includes two $\mathrm{NiO}_{6}$ clusters with intercluster coupling between the Ni- $d$ and ligand states. The details of the model construction can be found in Ref. [31].

The parameters for the single-particle part of the Hamiltonian in our calculation are derived from downfolded values using the Stuttgart-NMTO program $[63,64]$. Following the same convention of Ref. [31], we define the parameters in the nondisproportionated state as $V_{e g}=2.8$, $V_{t 2 g}=1.5,10 D q=0.9$, and $T_{p p}=0.5$, all in units of eV. The monopole Coulomb interaction within the $d$ shell is set to $U_{d d}=6.0 \mathrm{eV}$ and, between the $d$ and core $p$ shells, to $U_{p d}=7.0 \mathrm{eV}$. The multipole part is set to $80 \%$ of the Hartree-Fock values of the Slater integrals [65]. The charge-transfer energy is $\Delta=-1.0 \mathrm{eV}$. For the bondordered state, the disproportionation value $\delta d=d_{\mathrm{Ni}-\mathrm{O}}^{A}-$ $d_{\mathrm{Ni}-\mathrm{O}}^{B}$ is defined as the deviation of the average bond length $d_{\mathrm{Ni}-\mathrm{O}}^{A(B)}$ of the expanded $A$ (compressed $B$ ) $\mathrm{NiO}_{6}$ octahedra from their mean value. For the $\mathrm{NdNiO}_{3}$ film used in this study, $\delta=0.04 \AA$ is determined by experiments [42]. The intercluster mixing is set to 0.33 [31] to reproduce the experimental energy splitting between $A$ and $B$.

We note that the relative intensity of the resonance $A$ with respect to $B$ is slightly higher in the experiment than in the calculation. This has also been observed in previous studies [66] and may be an indication of an additional $\mathrm{Ni}^{2+}$ contribution from oxygen off-stoichiometry at the sample surface.

For the RIXS spectra measured at $A$, the $d d$ excitation around $1 \mathrm{eV}$ and its change of line shape across the bond ordering transition is well reproduced. For the RIXS measured at $B$, the fluorescence intensity around $2-3 \mathrm{eV}$ dominates the spectral weight, similar to the experimental observation. For both incident energies, the calculated RIXS spectra show broad charge-transfer spectral weight centered around $4 \mathrm{eV}$, again in good agreement with the experimental data.

Figure 5 shows the site-resolved XAS and XMCD spectra calculated using the above-mentioned double-cluster model. The energies $E_{A}$ and $E_{B}$ are marked with vertical dashed lines
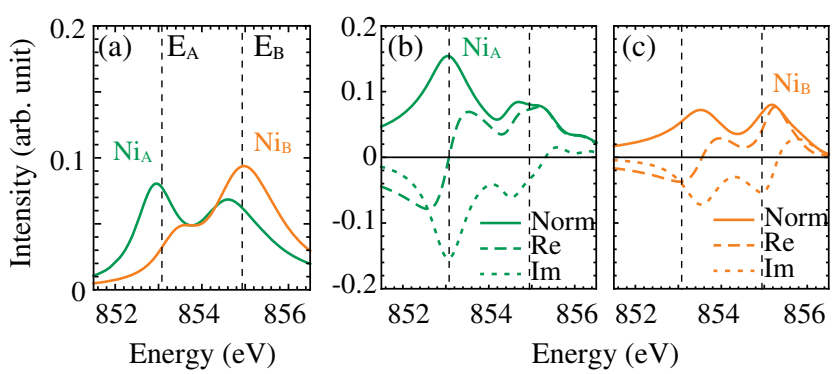

FIG. 5. Site-resolved (a) XAS and (b,c) XMCD spectra described through the complex circular dichroic form factor $\sigma^{(1)}$ [50] calculated using the double-cluster model. The spectra for the expanded and compressed $\mathrm{Ni}$ sites are plotted in green and orange, respectively. The two vertical dashed lines mark the energies $E_{A}$ (left) and $E_{B}$ (right) within each panel.

in each panel. For the XAS, the expanded and compressed $\mathrm{Ni}$ sites contribute dominantly to peaks $A$ and $B$, respectively. The XMCD spectra show a similar site dependence as the XAS. The energy-dependent coupling to spin excitations in RIXS can be estimated by the norm of the XMCD spectra, which shows a maximum close to $E_{A}$ and $E_{B}$ for the expanded and compressed sites, respectively.

\section{APPENDIX B: MAGNETIC MODEL AND SPIN-WAVE CALCULATION}

Considering small fluctuations of spins near the classical configuration in Fig. 4(a), we derive the magnon dispersions within the $J_{1}-J_{2}-J_{4}$ model, supplemented by a singleion anisotropy term $-K S_{\alpha}^{2}$, where $\alpha$ refers to the easy axis directions assumed to be oriented along the cubic body diagonals (as suggested by experiments [36-39]). The result reads as

$$
\omega_{ \pm}(\boldsymbol{q})=3 \sqrt{X_{\boldsymbol{q}} Y_{\boldsymbol{q}}\left[\left(1+\delta^{2}\right) \pm R_{\boldsymbol{q}}\right]},
$$

where the subscript $+(-)$ denotes the upper (lower) branch of the dispersion. The short-hand notations here are

$$
\begin{aligned}
X_{q} & =2 J_{2} \eta_{\boldsymbol{q}}+J_{4}\left(1-\gamma_{2 q}\right)+K / 3, \\
Y_{\boldsymbol{q}} & =2 J_{2} \xi_{\boldsymbol{q}}+J_{4}\left(1+\gamma_{2 q}\right)+K / 3, \\
R_{\boldsymbol{q}} & =\sqrt{(2 \delta)^{2}+\lambda_{q}^{2}},
\end{aligned}
$$

with

$$
\begin{aligned}
& \eta_{\boldsymbol{q}}=\frac{1}{3}\left(\sin q_{x} \sin q_{y}+\sin q_{y} \sin q_{z}+\sin q_{z} \sin q_{x}\right), \\
& \xi_{\boldsymbol{q}}=\frac{1}{3}\left(\cos q_{x} \cos q_{y}+\cos q_{y} \cos q_{z}+\cos q_{z} \cos q_{x}\right), \\
& \gamma_{\boldsymbol{q}}=\frac{1}{3}\left(\cos q_{x}+\cos q_{y}+\cos q_{z}\right), \\
& \lambda_{\boldsymbol{q}}=\left(1-\delta^{2}\right) \frac{J_{1} \gamma_{q}}{Y_{\boldsymbol{q}}} .
\end{aligned}
$$


At the $J_{1}=0$ limit, $\omega_{+}$and $\omega_{-}$correspond to the independent magnon dispersions within the $S_{A}$ and $S_{B}$ sublattices, respectively. The stability condition $\omega_{ \pm}>0$ requires $\left|J_{1}\right|<J_{1 c}=2 \sqrt{2 K\left(J_{2}+J_{4}\right) / 3}$. For $\left|J_{1}\right| \geq J_{1 c}$, the magnetic structure becomes incommensurate at $\boldsymbol{q}=\mathbf{Q}\left\{1+[1 /(2 \pi)]\left[J_{1} /\left(J_{2}+J_{4}\right)\right]\right\}$.

To calculate the magnetic RIXS intensity, the RIXS operator is approximated as $R_{A(B)}=\sigma_{A(B)}^{(1)}\left(\boldsymbol{\epsilon}_{i} \times \boldsymbol{\epsilon}_{o}^{*}\right) \cdot \mathbf{S}_{A(B)}$, with $\boldsymbol{\epsilon}_{i(o)}$ the polarization of the incident (emitted) photons. The RIXS intensity for a given $\boldsymbol{q}$ can then be calculated as

$$
\begin{aligned}
I_{ \pm}(\boldsymbol{q})= & \frac{\omega_{ \pm}(\boldsymbol{q}+\mathbf{Q})}{3 X_{\boldsymbol{q}+\mathbf{Q}}} \\
& \times\left[\left(|\alpha|^{2} p_{x}^{2}+|\beta|^{2} p_{z}^{2}\right) \pm \frac{2 \delta}{R_{\boldsymbol{q}+\mathbf{Q}}}\left(|\alpha|^{2} p_{x}^{2}-|\beta|^{2} p_{z}^{2}\right)\right] \\
& +\frac{3 X_{\boldsymbol{q}}}{\omega_{ \pm}(\boldsymbol{q})} p_{y}^{2}\left\{\left(|\tilde{\alpha}|^{2}+|\tilde{\beta}|^{2}\right) \pm\right. \\
& \left.\times \frac{1}{R_{\boldsymbol{q}}}\left[2\left(|\tilde{\alpha}|^{2}-|\tilde{\beta}|^{2}\right) \delta+\left(\tilde{\alpha}^{*} \tilde{\beta}+\tilde{\alpha} \tilde{\beta}^{*}\right) \lambda_{\boldsymbol{q}}\right]\right\}
\end{aligned}
$$

for the two branches. Here, $\tilde{\alpha}=\alpha(1+\delta)$ and $\tilde{\beta}=\beta(1-\delta)$; $\boldsymbol{p}=\left(p_{x}, p_{y}, p_{z}\right)$ is the orientation vector $\boldsymbol{\epsilon}_{i} \times \boldsymbol{\epsilon}_{o}^{*}$; and $\alpha$ and $\beta$ are the calculated (complex) values of $\sigma^{(1)}$ for sites $A$ and $B$ for a given incident energy, as discussed in Appendix A. For each $\boldsymbol{q}$ value, the RIXS intensity is calculated by averaging over the four $h k$ domains as

$$
\begin{aligned}
\bar{I}_{ \pm}\left(q_{x}, q_{y}, q_{z}\right)= & \frac{1}{4}\left[I_{ \pm}\left(q_{x}, q_{y}, q_{z}\right)+I_{ \pm}\left(-q_{x}, q_{y}, q_{z}\right)\right. \\
& \left.+I_{ \pm}\left(q_{x},-q_{y}, q_{z}\right)+I_{ \pm}\left(-q_{x},-q_{y}, q_{z}\right)\right] .
\end{aligned}
$$

Finally, the intensity is averaged over emission polarizations $\boldsymbol{\epsilon}_{o}$, both parallel and perpendicular to the scattering plane, as in the experiment we do not resolve the polarization of the scattered photons.

[1] H. Y. Hwang, Y. Iwasa, M. Kawasaki, B. Keimer, N. Nagaosa, and Y. Tokura, Emergent Phenomena at Oxide Interfaces, Nat. Mater. 11, 103 (2012).

[2] D. J. Scalapino, A Common Thread: The Pairing Interaction for Unconventional Superconductors, Rev. Mod. Phys. 84, 1383 (2012).

[3] M. Bibes and A. Barthelemy, Oxide Spintronics, IEEE Trans. Electron Devices 54, 1003 (2007).

[4] H. Béa, M. Gajek, M. Bibes, and A. Barthélémy, Spintronics with Multiferroics, J. Phys. Condens. Matter 20, 434221 (2008).

[5] M. Bibes, J. E. Villegas, and A. Barthelemy, Ultrathin Oxide Films and Interfaces for Electronics and Spintronics, Adv. Phys. 60, 5 (2011).
[6] H. Yamada, Y. Ogawa, Y. Ishii, H. Sato, M. Kawasaki, H. Akoh, and Y. Tokura, Engineered Interface of Magnetic Oxides, Science 305, 646 (2004).

[7] K.-I. Kobayashi, T. Kimura, H. Sawada, K. Terakura, and Y. Tokura, Room-Temperature Magnetoresistance in an Oxide Material with an Ordered Double-Perovskite Structure, Nature (London) 395, 677 (1998).

[8] L. J. P. Ament, M. van Veenendaal, T. P. Devereaux, J. P. Hill, and J. van den Brink, Resonant Inelastic X-ray Scattering Studies of Elementary Excitations, Rev. Mod. Phys. 83, 705 (2011).

[9] L. Braicovich, J. van den Brink, V. Bisogni, M. M. Sala, L. J. P. Ament, N. B. Brookes, G. M. De Luca, M. Salluzzo, T. Schmitt, V. N. Strocov, and G. Ghiringhelli, Magnetic Excitations and Phase Separation in the Underdoped $\mathrm{La}_{2-x} \mathrm{Sr}_{x} \mathrm{CuO}_{4}$ Superconductor Measured by Resonant Inelastic X-ray Scattering, Phys. Rev. Lett. 104, 077002 (2010).

[10] M. Le Tacon, G. Ghiringhelli, J. Chaloupka, M. M. Sala, V. Hinkov, M. W. Haverkort, M. Minola, M. Bakr, K. J. Zhou, S. Blanco-Canosa, C. Monney, Y. T. Song, G. L. Sun, C. T. Lin, G. M. De Luca, M. Salluzzo, G. Khaliullin, T. Schmitt, L. Braicovich, and B. Keimer, Intense Paramagnon Excitations in a Large Family of High-Temperature Superconductors, Nat. Phys. 7, 725 (2011).

[11] M. P. M. Dean, G. Dellea, R. S. Springell, F. Yakhou-Harris, K. Kummer, N. B. Brookes, X. Liu, Y.-J. Sun, J. Strle, T. Schmitt, L. Braicovich, G. Ghiringhelli, I. Bozovic, and J. P. Hill, Persistence of Magnetic Excitations in $\mathrm{La}_{2-x} \mathrm{Sr}_{x} \mathrm{CuO}_{4}$ from the Undoped Insulator to the Heavily Overdoped Nonsuperconducting Metal, Nat. Mater. 12, 1019 (2013).

[12] W. S. Lee, J. J. Lee, E. A. Nowadnick, S. Gerber, W. Tabis, S. W. Huang, V. N. Strocov, E. M. Motoyama, G. Yu, B. Moritz, H. Y. Huang, R. P. Wang, Y. B. Huang, W. B. Wu, C. T. Chen, D. J. Huang, M. Greven, T. Schmitt, Z. X. Shen, and T. P. Devereaux, Asymmetry of Collective Excitations in Electron- and Hole-Doped Cuprate Superconductors, Nat. Phys. 10, 883 (2014).

[13] J. Schlappa, K. Wohlfeld, K. J. Zhou, M. Mourigal, M. Haverkort, V. N. Strocov, L. Hozoi, C. Monney, S. Nishimoto, S. Singh, A. Revcolevschi, J. S. Caux, L. Patthey, H. M. Rønnow, J. van den Brink, and T. Schmitt, Spin-Orbital Separation in the Quasi-One-Dimensional Mott Insulator $\mathrm{Sr}_{2} \mathrm{CuO}_{3}$, Nature (London) 485, 82 (2012).

[14] J. Kim, M. Daghofer, A. H. Said, T. Gog, J. van den Brink, G. Khaliullin, and B. J. Kim, Excitonic Quasiparticles in a Spin-Orbit Mott Insulator, Nat. Commun. 5, 4453 (2014).

[15] G. Fabbris, D. Meyers, L. Xu, V. M. Katukuri, L. Hozoi, X. Liu, Z.-Y. Chen, J. Okamoto, T. Schmitt, A. Uldry, B. Delley, G. D. Gu, D. Prabhakaran, A. T. Boothroyd, J. van den Brink, D. J. Huang, and M. P. M. Dean, Doping Dependence of Collective Spin and Orbital Excitations in the Spin-1 Quantum Antiferromagnet $\mathrm{La}_{2-x} \mathrm{Sr}_{x} \mathrm{NiO}_{4}$ Observed by X Rays, Phys. Rev. Lett. 118, 156402 (2017).

[16] M. P. M. Dean, R. S. Springell, C. Monney, K. J. Zhou, J. Pereiro, I. Božović, B. Dalla Piazza, H. M. Rønnow, E. Morenzoni, J. van den Brink, T. Schmitt, and J. P. Hill, Spin Excitations in a Single $\mathrm{La}_{2} \mathrm{CuO}_{4}$ Layer, Nat. Mater. 11, 850 (2012). 
[17] G. Ghiringhelli, G. Dellea, L. Braicovich, M. Minola, M. Le Tacon, F. Baiutti, G. Cristiani, G. Logvenonv, B. Keimer, and M. Salluzzo, RIXS-Probed Spin Excitations in One Individual Unit Cell of "214" and "123" Cuprate Superconductors, APS Meeting Abstracts, 2014, http://adsabs .harvard.edu/abs/2014APS..MARG52006G.

[18] D. Betto, Y. Y. Peng, S. B. Porter, G. Berti, A. Calloni, G. Ghiringhelli, and N.B. Brookes, Three-Dimensional Dispersion of Spin Waves measured in NiO by Resonant Inelastic X-Ray Scattering, Phys. Rev. B 96, 020409 (2017); S. G. Chiuzbăian, G. Ghiringhelli, C. Dallera, M. Grioni, P. Amann, X. Wang, L. Braicovich, and L. Patthey, Localized Electronic Excitations in NiO Studied with Resonant Inelastic X-ray Scattering at the Ni M Threshold: Evidence of Spin Flip, Phys. Rev. Lett. 95, 197402 (2005); G. Ghiringhelli, A. Piazzalunga, C. Dallera, T. Schmitt, V. N. Strocov, J. Schlappa, L. Patthey, X. Wang, H. Berger, and M. Grioni, Observation of Two Nondispersive Magnetic Excitations in NiO by Resonant Inelastic Soft-X-ray Scattering, Phys. Rev. Lett. 102, 027401 (2009).

[19] J. B. Torrance, P. Lacorre, A. I. Nazzal, E. J. Ansaldo, and C. Niedermayer, Systematic Study of Insulator-Metal Transitions in Perovskites $\mathrm{RNiO}_{3}(\mathrm{R}=\mathrm{Pr}, \mathrm{Nd}, \mathrm{Sm}, \mathrm{Eu})$ Due to Closing of Charge-Transfer Gap, Phys. Rev. B 45, 8209 (1992).

[20] J. L. García-Muñoz, J. Rodríguez-Carvajal, and P. Lacorre, Neutron-Diffraction Study of the Magnetic Ordering in the Insulating Regime of the Perovskites $\mathrm{RNiO}_{3}(\mathrm{R}=\mathrm{Pr})$ and Nd, Phys. Rev. B 50, 978 (1994).

[21] G. Catalan, Progress in Perovskite Nickelate Research, Phase Transitions 81, 729 (2008).

[22] J.-S. Zhou, J. B. Goodenough, B. Dabrowski, P. W. Klamut, and Z. Bukowski, Enhanced Susceptibility in $\mathrm{LNiO}_{3}$ Perovskites ( $\mathrm{L}=\mathrm{La}, \operatorname{Pr}, \mathrm{Nd}, \mathrm{Nd}_{0.5} \mathrm{Sm}_{0.5}$ ), Phys. Rev. Lett. 84, 526 (2000).

[23] S. Lee, R. Chen, and L. Balents, Metal-Insulator Transition in a Two-Band Model for the Perovskite Nickelates, Phys. Rev. B 84, 165119 (2011).

[24] Y. Lu, Z. Zhong, M. W. Haverkort, and P. Hansmann, Origins of Bond and Spin Order in Rare-Earth Nickelate Bulk and Heterostructures, Phys. Rev. B 95, 195117 (2017).

[25] I. I. Mazin, D. I. Khomskii, R. Lengsdorf, J. A. Alonso, W. G. Marshall, R. M. Ibberson, A. Podlesnyak, M. J. Martínez-Lope, and M. M. Abd-Elmeguid, Charge Ordering as Alternative to Jahn-Teller Distortion, Phys. Rev. Lett. 98, 176406 (2007).

[26] M. Medarde, P. Lacorre, K. Conder, F. Fauth, and A. Furrer, Giant ${ }^{16} \mathrm{O}-{ }^{18} \mathrm{O}$ Isotope Effect on the Metal-Insulator Transition of $\mathrm{RNiO}_{3}$ Perovskites ( $\mathrm{R}=$ Rare) Earth, Phys. Rev. Lett. 80, 2397 (1998).

[27] R. Jaramillo, S. D. Ha, D. Silevitch, and S. Ramanathan, Origins of Bad-Metal Conductivity and the Insulator-Metal Transition in the Rare-Earth Nickelates, Nat. Phys. 10, 304 (2014).

[28] G. G. Guzmán-Verri, R. T. Brierley, and P. B. Littlewood, Elastic Interactions and Control of the Mott Transition, arXiv: 1701.02318.

[29] J. Zaanen, G. A. Sawatzky, and J. W. Allen, Band Gaps and Electronic Structure of Transition-Metal Compounds, Phys. Rev. Lett. 55, 418 (1985).
[30] S. Johnston, A. Mukherjee, I. Elfimov, M. Berciu, and G. A. Sawatzky, Charge Disproportionation without Charge Transfer in the Rare-Earth-Element Nickelates as a Possible Mechanism for the Metal-Insulator Transition, Phys. Rev. Lett. 112, 106404 (2014).

[31] R. J. Green, M. W. Haverkort, and G. A. Sawatzky, Bond Disproportionation and Dynamical Charge Fluctuations in the Perovskite Rare-Earth Nickelates, Phys. Rev. B 94, 195127 (2016).

[32] H. Park, A. J. Millis, and C. A. Marianetti, Site-Selective Mott Transition in Rare-Earth-Element Nickelates, Phys. Rev. Lett. 109, 156402 (2012).

[33] H. Park, A. J. Millis, and C. A. Marianetti, Total Energy Calculations Using DFT + DMFT: Computing the Pressure Phase Diagram of the Rare Earth Nickelates, Phys. Rev. B 89, 245133 (2014).

[34] B. Lau and A.J. Millis, Theory of the Magnetic and Metal-Insulator Transitions in $\mathrm{RNiO}_{3}$ Bulk and Layered Structures, Phys. Rev. Lett. 110, 126404 (2013).

[35] A. Subedi, O. E. Peil, and A. Georges, Low-Energy Description of the Metal-Insulator Transition in the Rare-Earth Nickelates, Phys. Rev. B 91, 075128 (2015).

[36] M. T. Fernández-Díaz, J. A. Alonso, M. J. Martínez-Lope, M. T. Casais, and J. L. García-Muñoz, Magnetic Structure of the $\mathrm{HoNiO}_{3}$ Perovskite, Phys. Rev. B 64, 144417 (2001).

[37] V. Scagnoli, U. Staub, A. M. Mulders, M. Janousch, G. I. Meijer, G. Hammerl, J. M. Tonnerre, and N. Stojic, Role of Magnetic and Orbital Ordering at the Metal-Insulator Transition in $\mathrm{NdNiO}_{3}$, Phys. Rev. B 73, 100409 (2006).

[38] V. Scagnoli, U. Staub, Y. Bodenthin, M. García-Fernández, A. M. Mulders, G. I. Meijer, and G. Hammerl, Induced Noncollinear Magnetic Order of $\mathrm{Nd}^{3+}$ in $\mathrm{NdNiO}_{3}$ Observed by Resonant Soft X-ray Diffraction, Phys. Rev. B 77, 115138 (2008).

[39] A. Frano, E. Schierle, M. W. Haverkort, Y. Lu, M. Wu, S. Blanco-Canosa, U. Nwankwo, A. V. Boris, P. Wochner, G. Cristiani, H. U. Habermeier, G. Logvenov, V. Hinkov, E. Benckiser, E. Weschke, and B. Keimer, Orbital Control of Noncollinear Magnetic Order in Nickel Oxide Heterostructures, Phys. Rev. Lett. 111, 106804 (2013).

[40] J. Ruppen, J. Teyssier, O. E. Peil, S. Catalano, M. Gibert, J. Mravlje, J.-M. Triscone, A. Georges, and D. van der Marel, Optical Spectroscopy and the Nature of the Insulating State of Rare-Earth Nickelates, Phys. Rev. B 92, 155145 (2015).

[41] N. B. Brookes (unpublished).

[42] Y. Lu, A. Frano, M. Bluschke, M. Hepting, S. Macke, J. Strempfer, P. Wochner, G. Cristiani, G. Logvenov, H.-U. Habermeier, M. W. Haverkort, B. Keimer, and E. Benckiser, Quantitative Determination of Bond Order and Lattice Distortions in Nickel Oxide Heterostructures by Resonant X-ray Scattering, Phys. Rev. B 93, 165121 (2016).

[43] See Supplemental Material at http://link.aps.org/ supplemental/10.1103/PhysRevX.8.031014 for details of transport measurement, RIXS raw data, and magnetic spectra along [101].

[44] V. Bisogni, S. Catalano, R. J. Green, M. Gibert, R. Scherwitzl, Y. Huang, V. N. Strocov, P. Zubko, S. Balandeh, J.-M. Triscone, G. Sawatzky, and T. Schmitt, Ground-State Oxygen Holes and the Metal-Insulator Transition in the Negative 
Charge-Transfer Rare-Earth Nickelates, Nat. Commun. 7, 13017 (2016).

[45] T. Mizokawa, H. Namatame, A. Fujimori, K. Akeyama, H. Kondoh, H. Kuroda, and N. Kosugi, Origin of the Band Gap in the Negative Charge-Transfer-Energy Compound $\mathrm{NaCuO}_{2}$, Phys. Rev. Lett. 67, 1638 (1991).

[46] T. Mizokawa, D. I. Khomskii, and G. A. Sawatzky, Spin and Charge Ordering in Self-Doped Mott Insulators, Phys. Rev. B 61, 11263 (2000).

[47] M. W. Haverkort, M. Zwierzycki, and O. K. Andersen, Multiplet Ligand-Field Theory Using Wannier Orbitals, Phys. Rev. B 85, 165113 (2012).

[48] Y. Lu, M. Höppner, O. Gunnarsson, and M. W. Haverkort, Efficient Real-Frequency Solver for Dynamical Mean-Field Theory, Phys. Rev. B 90, 085102 (2014).

[49] M. W. Haverkort, G. Sangiovanni, P. Hansmann, A. Toschi, Y. Lu, and S. Macke, Bands, Resonances, Edge Singularities and Excitons in Core Level Spectroscopy Investigated within the Dynamical Mean-Field Theory, Europhys. Lett. 108, 57004 (2014).

[50] M. W. Haverkort, Theory of Resonant Inelastic X-ray Scattering by Collective Magnetic Excitations, Phys. Rev. Lett. 105, 167404 (2010).

[51] T. P. Devereaux, A. M. Shvaika, K. Wu, K. Wohlfeld, C. J. Jia, Y. Wang, B. Moritz, L. Chaix, W.-S. Lee, Z.-X. Shen, G. Ghiringhelli, and L. Braicovich, Directly Characterizing the Relative Strength and Momentum Dependence of Electron-Phonon Coupling Using Resonant Inelastic X-ray Scattering, Phys. Rev. X 6, 041019 (2016).

[52] M. Hepting, M. Minola, A. Frano, G. Cristiani, G. Logvenov, E. Schierle, M. Wu, M. Bluschke, E. Weschke, H.-U. Habermeier, E. Benckiser, M. Le Tacon, and B. Keimer, Tunable Charge and Spin Order in $\mathrm{PrNiO}_{3}$ Thin Films and Superlattices, Phys. Rev. Lett. 113, 227206 (2014).

[53] V. Bisogni, L. Simonelli, L. J. P. Ament, F. Forte, M. Moretti Sala, M. Minola, S. Huotari, J. van den Brink, G. Ghiringhelli, N. B. Brookes, and L. Braicovich, Bimagnon Studies in Cuprates with Resonant Inelastic $X$-ray Scattering at the $O K$ Edge. I. Assessment on $\mathrm{La}_{2} \mathrm{CuO}_{4}$ and Comparison with the Excitation at $\mathrm{Cu} \mathrm{L}_{3}$ and $\mathrm{Cu} \mathrm{K}$ Edges, Phys. Rev. B 85, 214527 (2012).
[54] We note, however, that the charge excitations in RIXS are expected to be highly momentum dependent, and thus their contribution to the dispersionless spectra should be small.

[55] We note that the two data points of intensity 0.8 at $250 \mathrm{~K}$ are considered as noise. They cannot be an intrinsic feature of the spectrum because the width of the corresponding peak would be $15 \mathrm{meV}$, well below our instrumental resolution of $50 \mathrm{meV}$.

[56] J.-H. Kim, A. Jain, M. Reehuis, G. Khaliullin, D. C. Peets, C. Ulrich, J. T. Park, E. Faulhaber, A. Hoser, H. C. Walker, D. T. Adroja, A. C. Walters, D. S. Inosov, A. Maljuk, and B. Keimer, Competing Exchange Interactions on the Verge of a Metal-Insulator Transition in the Two-Dimensional Spiral Magnet $\mathrm{Sr}_{3} \mathrm{Fe}_{2} \mathrm{O}_{7}$, Phys. Rev. Lett. 113, 147206 (2014).

[57] C. Zener, Interaction between the $d$-shells in the Transition Metals. II. Ferromagnetic Compounds of Manganese with Perovskite Structure, Phys. Rev. 82, 403 (1951).

[58] P. W. Anderson and H. Hasegawa, Considerations on Double Exchange, Phys. Rev. 100, 675 (1955).

[59] P. G. de Gennes, Effects of Double Exchange in Magnetic Crystals, Phys. Rev. 118, 141 (1960).

[60] G. Khaliullin and R. Kilian, Theory of Anomalous Magnon Softening in Ferromagnetic Manganites, Phys. Rev. B 61, 3494 (2000).

[61] K. Nakajima, K. Yamada, S. Hosoya, T. Omata, and Y. Endoh, Spin-Wave Excitations in Two Dimensional Antiferromagnet of Stoichiometric $\mathrm{La}_{2} \mathrm{NiO}_{4}$, J. Phys. Soc. Jpn. 62, 4438 (1993).

[62] See http://www.diamond.ac.uk/Beamlines/Spectroscopy/ I21.html.

[63] O. K. Andersen and T. Saha-Dasgupta, Muffin-Tin Orbitals of Arbitrary Order, Phys. Rev. B 62, R16219 (2000).

[64] Y. Nohara and O. K. Andersen (unpublished).

[65] J. W. Freeland, M. van Veenendaal, and J. Chakhalian, Evolution of Electronic Structure across the Rare-Earth $\mathrm{RNiO}_{3}$ Series, J. Electron Spectrosc. Relat. Phenom. 208, 56 (2016).

[66] M. Medarde, A. Fontaine, J. L. García-Muñoz, J. RodríguezCarvajal, M. de Santis, M. Sacchi, G. Rossi, and P. Lacorre, $\mathrm{RNiO}_{3}$ Perovskites $(\mathrm{R}=\mathrm{Pr}, \mathrm{Nd})$ : Nickel Valence and the Metal-Insulator Transition Investigated by X-ray-Absorption Spectroscopy, Phys. Rev. B 46, 14975 (1992). 